\title{
First order phase transitions of spin systems*
}

\author{
Alain BILLOIRE ${ }^{\mathrm{a}}$ \\ aService de Physique Théorique de Saclay, \\ 91191 Gif sur Yvette France
}

I review some numerical ways to determine the parameters of systems close to a first order phase transition point: energy and specific heat of the coexisting phases and interface tension. Numerical examples are given for the 2-d $q$ states Potts model.

\section{INTRODUCTION}

First order phase transitions are easily described in terms of the competition between two phases (see e.g. [1]). Consider for definiteness a temperature driven phase transition between a disordered phase and a q-fold degenerate ordered phase of free energy $f_{d}(\beta)$ and $f_{o}(\beta)$. In order to use numerical methods, one needs to know the behavior of statistical averages in a finite volume, close to the transition point. This was more or less correctly guessed by several authors [2,3], and put on rigorous footing (at least for a class of models including the large $q$ Potts model) in Ref [4.5] where the partition function is shown to behave like

$$
\begin{aligned}
Z(\beta, L) & =e^{-L^{d} \beta f_{d}(\beta)}+q e^{-L^{d} \beta f_{o}(\beta)} \\
& +\mathcal{O}\left(e^{-b L}\right) e^{-\beta f(\beta) L^{d}} ; b>0
\end{aligned}
$$

This formula is correct for periodic boundary conditions, $f_{d}(\beta)$ and $f_{o}(\beta)$ are $L$ independent functions, and $f(\beta)=\min \left\{f_{d}(\beta), f_{o}(\beta)\right\}$. Although there is no analytical continuation of the free energy of one phase into the other phase, both $f_{d}(\beta)$ and $f_{o}(\beta)$ are shown to be several times differentiable at $\beta_{t}$, the (infinite volume) transition point where $f_{o}=f_{d}$. As a consequence the finite size behavior of any moment of the energy is obtained by differentiating Eq. 11. For

${ }^{*}$ Talk given at the XII International Symposium on Lattice Field Theory, LATTICE '94, Bielefeld Germany, September 27 - October 1 st 1994.

Preprint SPhT 94-164

hep-lat/9501003 example, the maximum of the energy fluctuation behaves like

$$
\left(<E^{2}>-<E>^{2}\right)_{\max }=\mathcal{A}+\mathcal{B} L^{-d}+\mathcal{O}\left(L^{-2 d}\right)(2)
$$

with similar results for $\left\langle S^{2}>-<S\right\rangle^{2}$, $B L=\frac{1}{3}\left(1-\frac{<E^{4}>}{\left.<E^{2}\right\rangle^{2}}\right)$ (the Binder Landau cumulant), $1-\frac{\left\langle E^{2}\right\rangle}{\langle E\rangle^{2}} \ldots$ The constants $\mathcal{A}$ and $\mathcal{B}$ in Eq.2 are simple functions of $E_{o}, E_{d}, C_{o}$ and $C_{d}$, the (infinite volume limit) energy and specific heat of the pure phases at $\beta_{t}$. Identical results for the first two terms in the $1 / L^{d}$ expansion can be obtained using the Binder Landau ansatz[3] for the energy probability density $P_{L}(E)$.

$$
\begin{aligned}
P_{L}(E) & =N\left[q \frac{a_{o}}{\sqrt{C_{o}}} e^{-\frac{L^{d} \beta_{t}^{2}\left(E-E_{o}-C_{o} \delta T\right)^{2}}{2 C_{o}}}\right. \\
& \left.+\frac{a_{d}}{\sqrt{C_{d}}} e^{-\frac{L^{d} \beta_{t}^{2}\left(E-E_{d}-C_{d} \delta T\right)^{2}}{2 C_{d}}}\right] \\
\delta T & =\frac{1}{\beta}-\frac{1}{\beta_{t}}
\end{aligned}
$$

which can be obtained from Eq.1 by inverse Laplace transform, neglecting the $\mathcal{O}\left(e^{-b L}\right)$ terms. In order to estimate $\mathcal{A}$ and $\mathcal{B}$ from numerical data, the presence of $1 / L^{2 d}$ corrections in Eq.2 is a nuisance. As first noticed in [5], averages estimated at $\beta_{t}$ are better behaved, for example the energy fluctuation

$$
\begin{aligned}
\left(<E^{2}>-<E>^{2}\right)_{\max } & =\tilde{\mathcal{A}}+\tilde{\mathcal{B}} L^{-d} \\
& +\mathcal{O}\left(e^{-b L}\right) ; b>0
\end{aligned}
$$


The energy itself behaves like,

$<E>_{L}\left(\beta_{t}\right)=<E>_{\infty}\left(\beta_{t}\right)+\mathcal{O}\left(e^{-b L}\right) ; b>0(5)$

A simple powerful method to determine the value for $\beta_{t}$ follows [5]: perform simulations on increasing sized lattices, at a value close to $\beta_{t}$. Extrapolate to neighboring values of $\beta$ using the Swendsen Wang reweighting method[6]. $\beta_{t}$ is determined as the fixed point of the set of curves $\left.\{<E\rangle_{L}(\beta)\right\}$. This is similar to Binder's cumulant method [7] to determine a second order phase transition point using the fourth order $\mathrm{cu}-$ mulant: $1-\frac{<S^{4}>}{\left.3<S^{2}\right\rangle^{2}}$. However in the current case, the estimate has only exponentially small corrections. Application $[\mathbb{1}$ of this method to the 2-d $q=10$ Potts model can be found in Fig.1. This is a strong first order phase transition (see Tab.11), the estimated value for $\beta_{t}$ is 1.42596(7) in excellent agreement with the exact value $\ln (\sqrt{10}+1) \sim 1.42606$.

\begin{tabular}{|lrrrr|}
\hline $\mathrm{q}$ & $C_{o}$ & $E_{d}-E_{o}[\sqrt[10]{10}$ & $C_{d}-C_{o}$ & $\xi_{\beta_{t}^{-}}[25]$ \\
\hline 5 & & 0.05292 & 0.0326 & 2512 \\
7 & $71.3(10)$ & 0.35328 & 0.2234 & 48.09 \\
10 & $18.06(4)$ & 0.69605 & .44763 & 10.56 \\
20 & $5.362(3)$ & 1.19416 & .77139 & 2.70 \\
\hline
\end{tabular}

Table 1

The 2-d Potts model: Values of $C_{o}$ from the large $q$ expansion, exact values for $E_{d}-E_{o}, C_{d}-C_{o}$, and correlation length $\xi\left(\beta \rightarrow \beta_{t}^{-}\right)$of the disordered phase. The correlation length of the ordered phase is smaller.

Comparison [9] of the predictions of Eq.2 and 5 with numerical data from lattices of size $16 \leq$ $L \leq 100$ can be found in Fig.2 2 and Fig. 3 for the 2 $\mathrm{d} q=10$ Potts model. In both figures the value of the specific heat $C V$ divided by $1 / L^{d}$ is plotted as function of $1 / L^{d}$. The value at $L=\infty$ is exactly known and the slope is related to the value of $C_{o}$ and $C_{d}$. An estimate using the large $q$ expansion of the model is 11 . $C_{o}=18.06(4)$. (The difference $C_{o}-C_{d}$ is exactly known). The numerical data

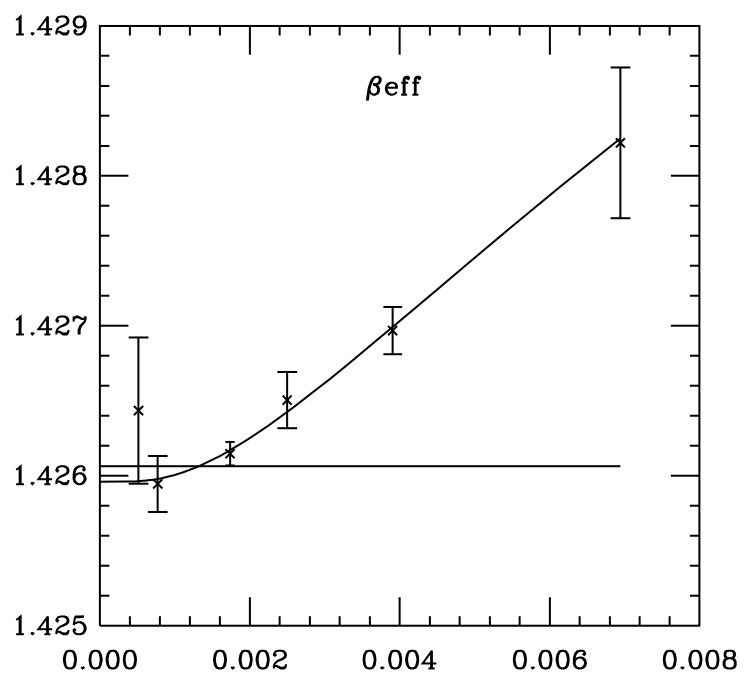

Figure 1. Estimate of the infinite volume transition temperature of the $q=10$ Potts model obtained from the equality of the internal energy of the following pairs of lattices: $\{12,16\},\{16,20\},\{20,24\},\{24,36\},\{36,44\},\{44,50\}$, as function of $1 / L^{2}$, where $L$ is the smallest value of each pair. The curve drawn is a fit to the shape $a+b e^{-c L}$. Note that the (unfounded) shape $a+b / L^{2}$ would give a good fit of the data but an incorrect estimate for $\beta_{t}$.

\begin{tabular}{|lll|}
\hline $\mathrm{q}$ & $C_{o}$ & reference \\
\hline 7 & $47.5(25)$ & 14 \\
& $50 .(10)$ & 8 \\
& $44 .(22)$ & 15 \\
\hline 10 & $10.6(11)$ & 16 \\
& $12.7(3)$ & \\
& $\sim 18$ & 13 \\
& $18.0(2)$ & 13 \\
\hline 15 & $8.04(4)$ & 13 \\
\hline 20 & $5.2(2)$ & 12 \\
& $5.38(4)$ & 13 \\
\hline
\end{tabular}

Table 2

Numerical estimates of the specific heat of the ordered phase of the $q$ states Potts model. Results are given in chronological order for each $q$ value. 


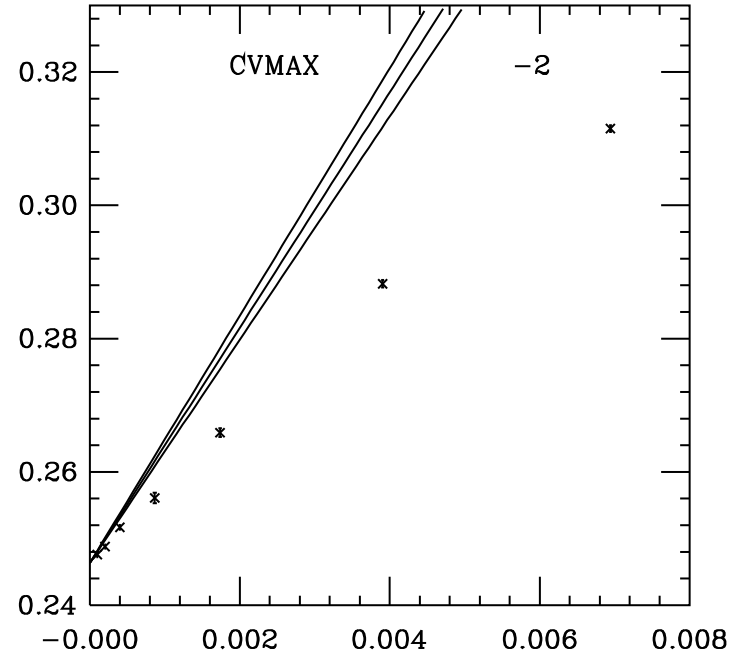

Figure 2. The maximum of the specific heat divided by $L^{2}$, as a function of $1 / L^{2}$, for the $q=10$ 2-d Potts model. The three curves are drawn using the values $C_{o}=18.06 \times\{.95,1.00,1.05\}$.

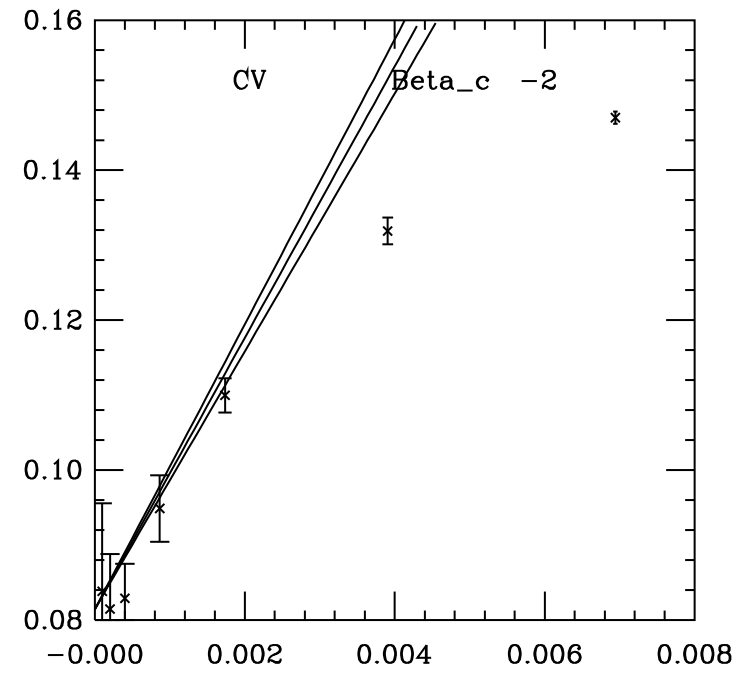

Figure 3. $C V\left(\beta_{t}\right) / L^{2}$, as a function of $1 / L^{2}$, for the $q=102$-d Potts model. The three curves are drawn using the values $C_{o}=18.06 \times$ $\{.95,1.00,1.05\}$. for $C V\left(\beta_{t}\right)$ would allow to estimate correctly the value for $C_{o}$. Using the data for $C V_{\max }$ or $B L_{\min }$ would however led to underestimate $C_{o}[9,8]$. For a very strong transition, like the one of the $q=20$ model, results (from similar sized lattices) are unambiguous [12]: both $C V\left(\beta_{t}\right), C V_{\max }$ and $B L_{\min }$ give estimates for $C_{o}$ that agree with the large $q$ result. On the other hand results for the $q=7$ model on lattices as large as $L=64$ do not enter the asymptotic regime 8 .

Published results for $C_{o}$ from such finite size analysis can be found in Tab. 1 for the $q=20$, 10 and 7 Potts models. The large $q$ expansion estimates can be found in Tab.1.

\section{DETERMINING THE ORDER OF A PHASE TRANSITION}

It is clear from the above that equations like $\mathrm{Eq} 2$ are only valid in the large volume limit $L>>\xi$. Marginal cases (the so called weak first order phase transitions) where $\xi$ is very large and the latent heat is very small are quite hard to discriminate from second order phase transitions, using numerical methods.

One signature for first order phase transition is the "two peak signal", namely

i) $P_{L}(E)$ has two peaks (of heights $P_{o}^{\max }$ and $\left.P_{d}^{\max }\right)$ with a minimum between them of height $P^{\text {min }}$.

ii) This structure behaves the way it should as $L$ grows, namely the distance between the two peaks goes to a constant and e.g. $P_{o}^{\max } / P^{\min } \rightarrow$ $\infty$.

The existence of two peaks in itself is not a signal of a first order phase transition. It is well known that, for example, the spin probability density of the Ising model has two peaks at the (infinite volume) transition temperature 17. The distance between the peaks goes to zero as $L$ grows as $L^{-\beta / \nu}$ (here $\beta$ is a critical exponent) and the ratio of the peak height to the minimum height stays constant.

The two peak signal is nearly as old as numerical simulations, it was made more precise in Ref. 18]: Define $\Delta F=\ln \left(P_{o}^{\max } / P^{\min }\right)$, measured at the transition point (at zero field for field driven transitions or at the transition tempera- 
ture for temperature driven transition.). If $\Delta F$ increases as $L$ grows the transition is first order, otherwise it is second order.

In the first order case $\Delta F$ behaves as $L^{d-1}$ for the following reason[7, 19]: Two phase configurations with almost planar interfaces dominate in the region around the minimum of $P_{L}(E)$. Let $E=x E_{o}+(1-x) E_{d}, x \in[0,1]$, the contribution of such configurations to the partition function is

$$
\begin{aligned}
Z^{\operatorname{mixed}}(x) & \varnothing \quad e^{-x L^{d} \beta f_{o}(\beta)} e^{-(1-x) L^{d} \beta f_{d}(\beta)} \\
& \times \quad L^{p} e^{-2 \sigma_{o, d} L^{d-1}} .
\end{aligned}
$$

The first two factors are contributions from the pure phases. The factor $L^{p} \exp \left(-2 \sigma_{o, d} L^{d-1}\right)$ comes from interface effects, $\sigma_{o, d}$ is the orderdisorder interface tension, and the exponent $p$ can be computed in a continuous approximation [20]). Finally $\Delta F \sim 2 \sigma_{o, d} L^{d-1}$ However in current simulations this behavior is only seen for extremely strong first order phase transitions, like the $q=20$ Potts model. To summarize, $\Delta F$ growing with $L$ is a signal for first order phase transition, $\Delta F \propto L^{d-1}$ is only seen for obviously first order cases.

It is a widespread belief that the behavior of $B L_{\text {min }}$ is a better signal for first order phase transitions that the behavior of $P_{L}(E)$ itself. $B L_{\min }$ has a non zero limit for first order transitions, and a zero limit for second order phase transitions or out of the precise transition point. Energy and spin fluctuations have similar properties. I would like to warn against this belief:

i) Suppose that, in the simulated range of lattice size, $P_{L}(E)$ is approximately $L$ independent, $B L_{\min }$ is then a non zero constant. Would any one conclude that the transition is first order? The only sensible conclusion is rather that one does not known the nature of the transition, and needs simulations on larger systems, in order to see wether $P_{L}(E)$ will shrink like for a second order transition, or will develop a two peak structure. Note that there do are examples in the literature of the use of $B L_{\min }$ to show that a transition is first order in cases where $P_{L}(E)$ is singled peaked.

ii) An example of the use of $B L_{\min }$ to deter- mine the order of the $2 \mathrm{~d} q \in[3,5]$ Potts model phase transitions can be found in Ref. 21] on lattices as large as $L=256$. $B L_{\min }$ does seem to go to a non zero limiting value for $q=5$ and to a zero limiting value for $q=3$ and 4 . The exact limiting value for $q=5$ is $B L_{\text {min }}=.44610^{-3}$ (called $V_{L, \min }-\frac{2}{3}$ in Fig.4 of Ref. 21]). Looking at the data, it means that there must be a crossover for $L>256$. The success of this method should thus be considered as somewhat accidental.

\section{INTERFACE TENSION}

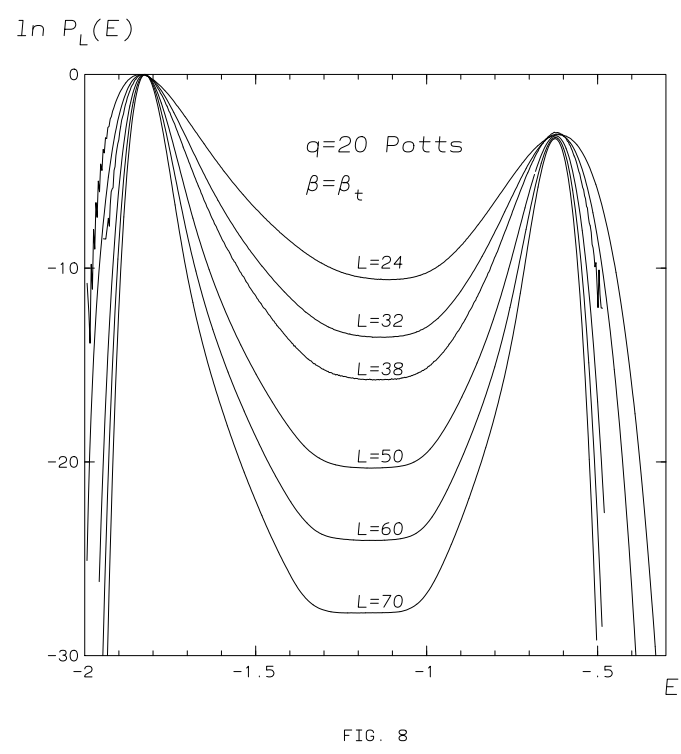

Figure 4. Plot of $\ln P_{L}\left(\beta_{t}, E\right)$ for selected lattice sizes in the $\mathrm{q}=20$ Potts model. The unfolding of a flat region with increasing lattice size $L>38$ is observed.

The best known technique to determine the order-disorder interface tension is the histogram method first introduced in 222. It uses the two peak structure of $P_{L}(E), \sigma_{o, d}$ is estimated as

$2 \sigma_{o, d}=\left.\frac{1}{2 L^{d-1}} \ln \left[\frac{P_{L}^{\max , o} P_{L}^{\max , d}}{\left(P_{L}^{\min }\right)^{2}}\right]\right|_{\beta=\beta_{t}}$

with $\mathcal{O}\left(1 / L^{d-1}\right)$ corrections. This method became a winner with the introduction of the multicanonical algorithm [23]. This algorithm brings 
two vast improvements: i) The exponentially large slowing down of the Metropolis algorithm is strongly reduced, presumably down to a power law slowing down. ii) The regions close to the peaks and the region close to the minimum are sampled equally. Fig. 4 shows our results 24 for the $q=20$ model energy probability distribution. One sees the unfolding of a flat region between the two peaks, in accordance with expectations (Eq.7 in $x$ independent at $\beta=\beta_{t}$ ). This unfolding has not (yet) been seen for lower values of $q$. Results for $\sigma_{o, d}$ obtained with this method are compared in Tab. 3 with the exact result [25] for various values of $q$. Also shown is an estimate using measurements of the auto-correlation time of the Swendsen-Wang algorithm [26], which behaves like $\tau \sim L^{\frac{d}{2}} e^{2 \sigma_{o, d} L^{d-1}}$ 277. This estimate is in qualitative agreement with the exact result. The following two comments are in order

i) It is somewhat surprising to obtain excellent agreement with the exact results for $q=10$ and $q=7$, since in both cases $P_{L}(E)$ does not unfolds its asymptotic shape.

ii) Ingenious methods [28,29] where two interfaces are created by setting one half of the system at a temperature $\beta_{t}+\delta \beta_{t}$ and the other half at a temperature $\beta_{t}-\delta \beta_{t}$ give results in strong disagreement with the exact result.

\begin{tabular}{|l|ll|}
\hline $\mathrm{q}$ & $2 \sigma_{o, d}$ & Reference \\
\hline 7 & $.1886(12)$ & 28 \\
& .20 & 29 \\
& $.0241(10)$ & 14 \\
& .020792 & exact \\
10 & .094701 & exact \\
& $.0978(8)$ & 23 \\
& $.0950(5)$ & 24 \\
15 & .239234 & exact \\
& $.263(9)$ & 26 \\
20 & .370988 & exact \\
& $.3714(13)$ & 24 \\
\hline
\end{tabular}

Table 3

Comparison of the numerical estimates of the order disorder interface tension of the 2-d Potts model with exact results.

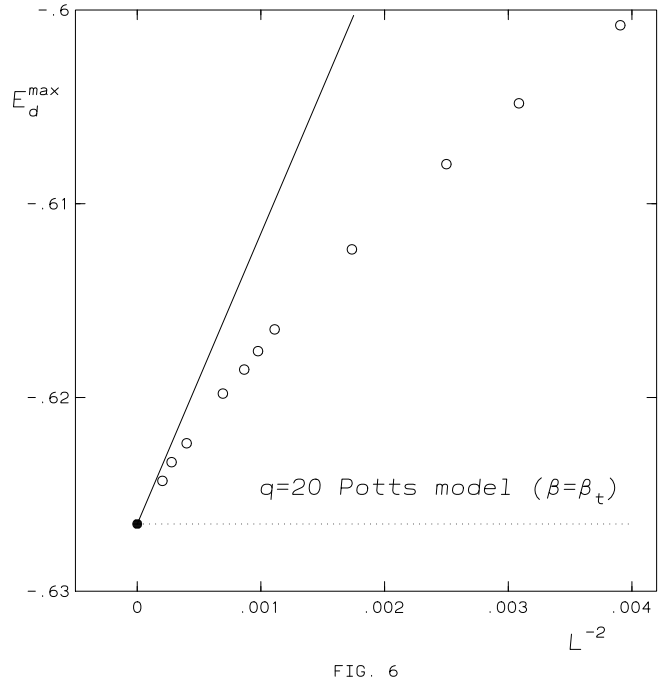

Figure 5. Locations of the maxima of the disordered peak $E_{L}^{\max , d}$ at $\beta_{t}$ in the $\mathrm{q}=20$ Potts model as function of the inverse volume.

Among the by products of interface tension measurements are precise determinations of the locations of the peaks of $P_{L}(E)$. These are seen to move with $L$, an effect not described by Eq.3. Two models have been proposed to explain this dependence. The first one 16 involves mixed phase effects, the prediction is $E_{o, d}^{\max }(L) \sim$ $E_{o, d}^{\max }(\infty)+A / L$. The second one involves higher order $1 / L^{d}$ terms in the Laplace transform of Eq.1, neglecting all $\mathcal{O}\left(e^{-b L}\right)$ terms, namely all mixed phase effects. The result is $E_{o, d}^{\max }(L) \sim$ $E_{o, d}^{\max }(\infty)+B / L^{d}$, where $B$ is related to the third derivative of the free energy of the phase. Numerical estimate 24 of $E_{d}^{\max }(L)$ as function of $1 / L^{d}$ are shown in Fig 5 for the $q=20$ model. The line drawn is the parameter free prediction of model two from Ref.[1] in leading order. Similar results are obtained for the $q=10$ case. The predicted $1 / L^{2}$ behavior is barely seen, but i) It has been shown in Ref.11 that the discrepancy can be accommodated by higher orders terms (still without any mixed phase effects) ii) A fit to the $1 / L$ form gives an estimates of $E_{d}^{\max }(\infty)$ incompatible with the exact value of $E_{d}$. 


\section{REFERENCES}

1. K. Binder and D. Heermann, Monte Carlo Simulations in Statistical Physics, Springer Series in Solid State Physics 80 (1988);

K. Binder, Rep. Prog. Phys. 50 (1987) 783;

H.J. Herrmann, W. Janke and F. Karsch (editors), Dynamics of First Order Phase Transitions, World Scientific, Singapore 1992.

2. K. Binder and D.P. Landau, Phys. Rev. B30 (1984) 1477.

3. M.S. Challa, D.P. Landau and K. Binder, Phys. Rev. B34 (1986) 1841.

4. C. Borgs and R. Kotecký, J. Stat. Phys. 61 (1990) 79 .

5. C. Borgs, R. Kotecký and S. Miracle-Sole, J. Stat. Phys. 62 (1991) 529.

6. A.M. Ferrenberg and R.H. Swendsen, Phys. Rev. Lett. 612635 (1988);

A.M. Ferrenberg and R.H. Swendsen, Phys. Rev. Lett. 631195 (1989).

7. K. Binder, Z. Phys. B43 119 (1981).

8. A. Billoire, R. Lacaze, and A. Morel, Nucl. Phys. B 370 (1992) 773.

9. A. Billoire and T. Neuhaus unpublished.

10. R.J. Baxter, J. Phys. A15, (1982) 3329;

R.J. Baxter, Exactly Solved Models in Statistical Mechanics, (Academic Press 1982).

11. T. Bhattacharya, R. Lacaze and A. Morel, Europh. Letters 83 (1993) 547;

Nucl. Phys. B (Proc. Supp.) 34 (1994) 671;

Saclay Preprint SPhT/94/008, To appear in Nucl. Phys. B;

Saclay Preprint SPhT/94/086, To appear.

12. A. Billoire, T. Neuhaus and B.A. Berg, Nucl. Phys. B396 (1993) 779.

13. W. Janke and S.K. Kappler, Nucl. Phys. B (proc. Sipp) 34 (1994) 674.

14. J. Janke, B. Berg and M. Katoot, Nucl. Phys. B382 (1992) 649.

15. K. Rummukainen, Nucl. Phys. B390 (1993) 621.

16. J. Lee and J.M. Kosterlitz, Phys. Rev. B43 (1991) 3265.

17. E. Eisenriegler, and R. Tomaschitz, Phys. Rev. B35 (1987) 4876.

18. J. Lee and J.M. Kosterlitz, Phys. Rev. Lett. 65 (1990) 137.
19. Commun. Math. Phys. 125 (1987) 81.

20. U. J. Wiese, Nucl. Phys. B375 (1992) 45;

M. Caselle, F. Gliozzi and S. Vinti, Phys. Lett B302 (1993) 74;

B. Bunk, Int. J. Mod. Phys. C3 (1992) 889.

21. M. Fukugita, H. Mino, M. Okawa and A. Ukawa, J. Phys. A 23 (1990) L561.

22. K. Binder, Phys. Rev. B25 (1982) 1699.

23. B.A. Berg and T. Neuhaus, Phys. Rev. Lett. 68 (1992) 9.

24. A. Billoire, T. Neuhaus and B.A. Berg, Nucl. Phys. B413 (1994) 795.

25. E. Buffenoir and S. Wallon, J. Phys. A26 (1993) 3045;

J. Johnson, S. Krinsky and B. Mc Coy, Phys. Rev. A8 (1973) 2526;

A. Klümper, A. Schadschneider and J. Zittartz, Z. Phys. B76 (1989) 247;

A. Klümper, Int. Journal of Mod. Phys. B4 (1990) 871;

C. Borgs and W. Janke, J. Phys. I (France) 2 (1992) 2011.

26. Sourendu Gupta, Phys. Lett. B325 (1994) 418.

27. JC. Niel and J. Zinn-Justin, Nucl. Phys. B280 (1987) 355.

28. J. Potvin and C. Rebbi, Phys. Rev. Lett 62 (1989) 3062;

S. Huang, J. Potvin, C. Rebbi and S. Sanielovici, Phys. Rev. D42 (1990) 2864.

29. A. Kajantie, L. Kärkkäinen and K. Rummukainen, Phys. Lett. 223B (1989) 213. 